\title{
Analysis of Ba Marker in Chinese
}

\author{
Lei Liu \\ Changchun University of Science and Technology, Changchun, China
}

\begin{abstract}
A large number of linguists have long been interested in ergativity, however ergative languages have long presented a vexing problem for them. The characteristics causing a language to be described as "ergative" is that the intransitive subject is marked in the same way as the transitive object morphologically, while the transitive subject receives a different case marking. Morphological case marking is the original criterion of ergativity. But, later, the term "syntactic ergativity" is used to characterize languages where syntactic phenomena treat the single argument of a verb like 'run' in the same way as the patient argument of a verb like 'hit', while the agent argument of this two-argument verb is treated differently. What about Chinese? Because scholars claim that in linguistics, Chinese is well known as an isolating or an analytic language which lacks case inflection or verb agreement, it is not morphologically ergative. In this paper, I'll first discuss Li and Yip's argument against the analysis of ba as an absolutive marker, then Fujii (1989)'s belief that the entire syntax is centred more or less round the absolutive in Chinese, and finally show that ba as an absolutive marker is unmotivated, rather than $b a$ should be treated as an object marker, sometimes optionally, sometimes obligatorily.
\end{abstract}

Index Terms - ba marker, ergativity, object marker

\section{INTRODUCTION}

A large number of linguists have long been interested in ergativity, however ergative languages have long presented a vexing problem for them. According to the overview articles written by Comrie(1978) and Dixon(1979) ${ }^{1}$, the characteristics causing a language to be described as "ergative" are that the intransitive subject is marked in the same way as the transitive object morphologically, while the transitive subject receives a different case marking. Morphological case marking is the original criterion of ergativity. But, later, the term "syntactic ergativity" is used to characterize languages where syntactic phenomena treat the single argument of a verb like 'run' in the same way as the patient argument of a verb like 'hit', while the agent argument of this two-argument verb is treated differently. Languages that have been described as ergative include Eskimo languages, Basque, some of the Polynesian languages, many Australian languages and Caucasian languages (Silva, 1989). What about Chinese ${ }^{2}$ ? Because scholars claim that in linguistics, Chinese is well known as an isolating or an analytic language which lacks case inflection or verb agreement, it is not morphologically ergative. But "it is possible for a language to be (wholly or partly) syntactically ergative in that it manifests syntactic phenomena which treat intransitive subjects and transitive objects alike, and in contrast to transitive subjects" (Li \& Yip, 1979, p.105). The function of $\boldsymbol{b} \boldsymbol{a}$ in modern Chinese is a widely discussed topic among linguists. Ba is treated as a verb (Hashimoto 1971), a preposition (coverb) (Travis, 1984; Li \& Thompson, 1981), or as a case marker (Huang, 1990). Henri Frei (1956), stimulated by Willem Grootaers (1953) who published an article on the analysis of $\boldsymbol{b} \boldsymbol{a}$, was the first scholar to recognize ergativity in Chinese, arguing that $\boldsymbol{b} \boldsymbol{a}$ should be treated as a marker of the absolutive case - the object of a transitive and the subject of an intransitive. We must admit that as far as the methodological accuracy and the depth of findings are concerned, Frei's investigation into $\boldsymbol{b} \boldsymbol{a}$ construction is said to be one of the most far-reaching explorations (Fujii, 1989). In addition, Fujii (1989) argues that in spite of the shortcoming of Frei's ergativity hypothesis which is based on distinguishing between the concepts of "inertial" and "energetic" only in semantic sense, he still believes that the entire syntax is centered more or less round the absolutive in Chinese. However, I fully agree with Li and Yip (1979) that $\boldsymbol{b} \boldsymbol{a}$ as a marker is not expected to be analyzed with respect to ergativity, because of two claims. One is that "it is shown that the very restricted number of cases where $\boldsymbol{b a}$ appears to mark an intransitive subject are all open to an alternative explanation", and second is "in any case, the very rarity of such cases by comparison with $\boldsymbol{b} \boldsymbol{a}$ on objects" (Li \& Yip, 1979, p.107). Furthermore, we can consider $\boldsymbol{b} \boldsymbol{a}$ only as an object marker (accusative). Actually, some direct objects are marked obligatorily preceded by the morpheme $\boldsymbol{b a}$, for other objects $\boldsymbol{b} \boldsymbol{a}$ is optional, or prohibited.

In this paper, I'll first discuss Li and Yip's argument against the analysis of $\boldsymbol{b} \boldsymbol{a}$ as an absolutive marker, then Fujii (1989)'s belief that the entire syntax is centered more or less round the absolutive in Chinese, and finally show that $\boldsymbol{b} \boldsymbol{a}$

\footnotetext{
${ }^{1}$ According to Song (2001) "in discussion of case marking within linguistic typology it has become a very useful convention to make reference to three grammatical-semantic primitives, A, S\&P. A stands for agent, or more accurately, the logical subject of the transitive clause; $\boldsymbol{P}$ for patient, or more accurately, the logical object of the transitive clause; $S$ is the sole argument, the logical subject of the transitive clause. "That is the criterion to judge the ergativity of a language.

2 "Mandarin Chinese," the official Chinese language, will simply be referred to as "Chinese" in this paper. In fact, it is slightly different from the dialect spoken in Hong Kong and in Taiwan.
} 
as an absolutive marker is unmotivated, rather than $\boldsymbol{b} \boldsymbol{a}$ should be treated as an object marker, sometimes optionally, sometimes obligatorily.

\section{AnAlysis Of BA AS AN ABSOlutive CASE MARKER}

It is claimed that the characteristics of " $\boldsymbol{b} \boldsymbol{a}$ marks specific direct objects dealt with transitive action verbs, but not all direct objects. Ba sentences must also include at least the aspect marker $\boldsymbol{l e}$, or resultative complement. It is subject to semantic and perhaps phonological restrictions ${ }^{3}$, the $\boldsymbol{b} \boldsymbol{a}$ NP having a definite referent" (Li \& Yip, 1979, p. 105).

\section{A. Criticism to Frei's Ba by Li and Yip (1979) and Fujii (1989)}

When linguistic typologists discuss case marking, it has become a very useful practice to make reference to "three grammatical-semantic primitives, A, S \& P. A stands for agent, or more accurately, the logical subject of the transitive clause; $\boldsymbol{P}$ for patient, or more accurately, the logical object of the transitive clause; $\boldsymbol{S}$ is the sole argument, the logical subject of the transitive clause" (Song, 2001, p.141). So in the ergative-absolutive system, $\boldsymbol{A}$ is identified with ergative case label, while $\boldsymbol{P}$ and $\boldsymbol{S}$ with absolutive. To put it differently, $\boldsymbol{S}$ and $\boldsymbol{P}$ are treated alike in contrast to $\boldsymbol{A}$. It is believed that ergative-absolutive case marking system is quite common though less common than nominative-accusative marking (Dixon, 1994, p.101; Song, 2001, p.141). Frei (1956) published an article in which he analyzed $\boldsymbol{b a}$ as an absolutive marker, because the intransitive subject $(\boldsymbol{S})$ is cased marked in the same way as the transitive object $(\boldsymbol{P})$ by $\boldsymbol{b} \boldsymbol{a}$ as a marker in the $\boldsymbol{b a}$-construction, while the transitive subject $(\boldsymbol{A})$ receives a different case marking. ${ }^{4}$ So, in order to prove that $\boldsymbol{b} \boldsymbol{a}$ is considered to be an ergative phenomenon, what is at issue is whether ba can mark intransitive $\operatorname{subject}(\boldsymbol{S})$, since it is well known that $\boldsymbol{b} \boldsymbol{a}$ marks objects. However, Li and Yip (1979) found that $\boldsymbol{b} \boldsymbol{a}$ marking of intransitive subjects is rare compared with $\boldsymbol{b} \boldsymbol{a}$ marking on the transitive objects. They classify Frei's examples of initial $\boldsymbol{b} \boldsymbol{a}$ into two groups and try to prove that these examples all have reasonable alternative explanations, that is to say, they don't carry the characteristics of $\boldsymbol{b} \boldsymbol{a}$ mark on the intransitive subject. The first group has "verbs which are normally or potentially transitive, and the sentences are interpreted as having anaphoric subjects, or as imperatives, depending on context" (Li \&Yip, 1979, p.105). The second group has verbs which are truly intransitive, such as pao (run) or zou (walk) or bing (ill). For example, (1) is an example of the first group and (2) \& (3)\&(4) of the second group.

(1) ba ta qi si le

ba S/He angry dead ASP

'S/he was made angry to death.' (adapted from Li and Yip1979)

(2) *Ba ge zhu pao le $e^{5}$

Ba CL pig run ASP

'A pig has run away.'

(3) *ba wo zou lei le

$\mathrm{Ba}$ I walk tired ASP

'I walk to feel tired.'

(4) *ba ta ba bing le

$\mathrm{Ba}$ his/her father ill ASP

'His father is ill.'

According to Li and Thompson (1981), we can argue that (1) can be treated as a $\boldsymbol{b} \boldsymbol{a}$ sentence without a subject when that subject refers to either a noun phrase or a proposition that is understood from the context. If the understood subject refers to a noun phrase, it is simply a case of a zero pronoun ${ }^{6}$. Consider example (5) from Frei (1371),

(5) a. Wo dong huai le

I freeze bad ASP

b. Ba wo dong huai le

$\mathrm{Ba}$ I freeze bad ASP

c. Dong huai le wo

Freeze bad ASP I

'I am freezing. '

According to common sense or context, we know we are freezing possibly because of the weather, so the subject is dummy "weather it" always realized as zero pronoun in Chinese. Therefore, $(5 \mathrm{~b})$ comes from the transitive (5c) which can also be expressed in the form of intransitive (5a). It is apparent $\boldsymbol{b} \boldsymbol{a}$ in (5b) marks the tansitive object, instead of intransitive subject, though $\boldsymbol{b a}$ is poistiond initially.

\footnotetext{
3 "Ba sentences must also include at least the aspect marker le, or other elaboration of the verb phrase such as frequency adverbials or an indirect object; this has been variously interpreted as a phonological constraint on monosyllabic verb phrases"(Li \&Yip, 1979). See details in Li (1974, p.455). ${ }^{4}$ In Chinese, actually, the ergative of ba construction is zero-marked, and the absolutive is marked by ba. In this case, Li and Yip (1979) believe that Chinese is the only case known to be zero-marked ergative, for David Nash points out that the established cases of ergative languages have ergative marked and absolutive unmarked (Nash, 1977, p. 87).

${ }^{5}$ This sentence selected by Li and Yip (1979) as an example, in fact, is not acceptable in modern Chinese, so here it cannot be used to prove that ba as an absolutive marker on intransitive subject. The same for the example (3) and (4)

${ }^{6} \mathrm{See} \mathrm{Li}$ and Thompson (1981) for detail in chapter 24.
} 
Sometimes the proposition is understood and doesn't need to be explicitly expressed, so if we want to discuss the way we treat a friend, we can simply say as in (1). Similarly, (3), if acceptable in Chinese, can be regarded as a $\boldsymbol{b} \boldsymbol{a}$ sentence without subject, and it can be interpreted in the sense that the walking the distance made me feel tired to the extent that I feel dead. Consider another example in (6),

(6) zuo-tian wo-men qing ke, ba ta chi de du-zi dou zhang le yesterday we $(\mathrm{pl})$ invite guest, ba s/he eat belly all bloat ASP

Yesterday we invite guests and that made him/her eat so much that his/her belly was bloated.

In this example the proposition is implied in the previous context (zuo- tian wo-men qing ke), it doesn't need to be overtly expressed in the ba sentence(ba ta chi de du -zi dou zhang le). This is the typical feature for the Chinese structure, with understood subject unexpressed directly.

The other two $\boldsymbol{b} \boldsymbol{a}$ sentences (2)and (4), though Li and Yip (1979) claim that they are the true examples of $\boldsymbol{b} \boldsymbol{a}$ marking intransitive subjects, actually, are not acceptable in modern Chinese, as a result, they cannot be treated as the typical example for $\boldsymbol{b} \boldsymbol{a}$ to mark intransitive subject. Besides (2) and (4), Li and Yip also list several examples to prove that there indeed exist examples of $\boldsymbol{b} \boldsymbol{a}$ marking intransitive subject. However, I find out that all of these examples are not acceptable in Chinese. Why? Frei's data is based on Cantonese or Mandarin spoken in Hong Kong. It is slightly different from the pu tong hua (Chinese) now officially used in China. That is why I found all of these examples proved ro be the true examples, are not acceptable according to pu tong hua in China. Consequently, so far, we cannot find a true example.

In a word, the question of whether $\boldsymbol{b} \boldsymbol{a}$ marks the intransitive subjects is crucial to Frei's analysis of $\boldsymbol{b} \boldsymbol{a}$ ergativity, but it turns out that examples (1)-(4) cannot be taken as convincing examples to illustrate that $\boldsymbol{b} \boldsymbol{a}$ marks the intansitive subject, for the reason that understood subject of $\boldsymbol{b} \boldsymbol{a}$ sentence is not necessarily overtly expressed.

$\mathrm{Li}$ and Yip also argue that if $\boldsymbol{b} \boldsymbol{a}$ is an absolutive marker, what is ergative marker? In Chinese, actually "the ergative of $\boldsymbol{b} \boldsymbol{a}$ construction is zero-marked, and the absolutive is marked by $\boldsymbol{b} \boldsymbol{a}$ ”. In this case, Li and Yip (1979) believe that Chinese is the only case known to be zero-marked ergative, for David Nash points out that the established cases of ergative languages have ergative marked and absolutive unmarked (Nash, 1977, p.87, from Li \& Yip, 1979).

As far as Frei is concerned, Chinese is not always ergative, exhibiting both the ergative-absolutive and nominativeaccusative based on "semantics" (Frei, 1956, p.83). Frei (1956) also says: "The use of ba presupposean ergative verb, whereas the latter does not presuppose $\boldsymbol{b a}$ "(p.96). In Li and Yip's interpretation, " $\boldsymbol{b} \boldsymbol{a}$ is an absolutive marker, but verbs may appear in either ergative /absolutive or nominative/accusative construction" (Li \&Yip, 1979, p.106). That is to say Chinese has the characteristics of "split ergativity". Frei therefore outlines the semantic conditions for ba, indicating that $\boldsymbol{b} \boldsymbol{a}$ NP must have a specific referent and an object of action verb in the case of transitive and he also observes that there is connection between $\boldsymbol{b} \boldsymbol{a}$ and aspect (either perfective le or a resultative complement).

For the split-ergative case marking system, the division between ergative/absolutive and nominative/accusative is "regular and systematic" (Song, 2001, p.148). One condition for division is "referential/semantic nature or the inherent lexical content of NPs" (Nominal Hierarchy (Dixon, 1994, p.85)). Another condition is "aspect and tense" with the language Georgian as example (Song, 2001, p.149. For example,

(7) a. student $-i \quad$ midis (from Song, 2001, p.149 Georgian)

Student-NOM goes

'The student goes.'

$\begin{array}{lll}\text { b. student }-i & \text { ceril-s } & \text { cers } \\ \text { Student-NOM } & \text { letter-ACC } & \text { writes }\end{array}$

'The student writes the letter.'

c. student $-i$ mivida Georgian (from Song, 2001, p.149 Georgian)

Student-ABS went

'The student went.'

d. student $-m a$ ceril-i dacera (from Song, 2001, p.149 Georgian)

Student-ERG letter-ABS wrote

'The student wrote the letter.'

(7a) and (7b) are in the present tense, and the suffix $-i$ is used to mark intransitive subject and transitive subject, in contrast to the suffix $-s$ in transitive object. On the other hand, (7c) and (7d) are in the ergative-absolutive case system because of the past tense (Song, 2001, p.149). It is not the case for Chinese ba sentense. Taking the conditions for split ergativity and semantic conditions for $\boldsymbol{b} \boldsymbol{a}$ into consideration, it is not natural for $\boldsymbol{b} \boldsymbol{a}$ to be analyzed with respect to split ergativity, for $\boldsymbol{b} \boldsymbol{a}$ sentence doesn't satisfy the conditions for split ergativity.

Moreover, in terms of Frei's ergativity hypothesis which is to distinguish "inertial" from "energetic", Fujii (1989) believed that it is best understood in the sense of the "active type" of language. What is "active type" of case system? In this system the case marking of intransitive subject depends on the semantic nature of the intransitive verb (Song, 2001, p.150). "If the intansitive verb refers to an activity which is likely to be under the control of subject, the subject will bear the same case marking as transitive verb subject", and "if it refers to non-controlled activity, the subject will be marked in the same way as transitive object" (Song, 2001, p. 150). Consider the example (8):

(8) a. Deng xi-mie le 
Light go out ASP

b. Ba deng xi-mie le (adapted from Grootaers, 16)

Ba lamp go out ASP

c. wo xie -mie le deng

I go out ASP lamp

d. wo ba deng xi-mie le

I ba lamp go out ASP

e. wo deng xi-mie le

I lamp go out ASP

'I turn off the lamp.'

Here in (8a) xi-mie, intransitive verb, refers to an activity which is not likely to be controlled by the subject (deng), so the subject (deng) will be marked in the same way as transitive object, thus (8b) (Ba deng xi mie le ) equals to (8d)( wo ba deng xi-mie le). But (8a) and (8b) are not identical in terms of meaning, and (8b) share the similar meaning with (8d) except (8b) has no subject, because it can infer from the context. In addition, xi-mie also behaves as transitive verb in (8c). In (8e), object (deng) is moved to preverbal position, but $\boldsymbol{b} \boldsymbol{a}$ is not added. Therefore how can we explain the sentences (8)? I, personally think $\boldsymbol{b} \boldsymbol{a}$ should be considered only as object marker. Some direct objects are marked obligatorily preceded by the morpheme $\boldsymbol{b} \boldsymbol{a}$; for some objects $\boldsymbol{b} \boldsymbol{a}$ is optional, and for some $\boldsymbol{b} \boldsymbol{a}$ is prohibited. In this way can we make a systematic analysis of the sentences (8). In the next section I will examine what factors influence the realization of the $\boldsymbol{b} \boldsymbol{a}$ as an object marker.

\section{B. Ba as Object Marker}

Chinese is well known as an isolating or an analytic language which lacks inflectional morphology. In other words, words in Chinese are generally not marked by any morphology showing their role in the sentence, so word order carries a lot of importance and the basic grammatical relations are identified by word order only. Chinese has unmarked word order-SVO.Consider example (9a):

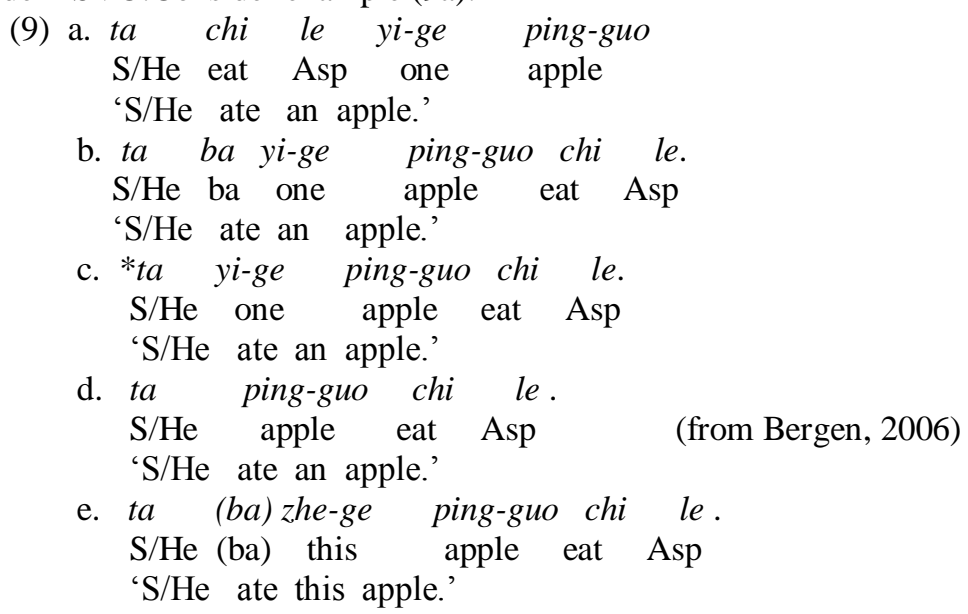

The subject $\boldsymbol{t} \boldsymbol{a}$ is positioned on the left side of the verb chi (eat), while direct object (yi-ge ping-guo) is placed on the right side of verb. Although word order in Chinese is rather strict, direct object can be moved to a preverbal position. Compare examples (9b) and (9c) and (9d), we can find that if the direct object is moved to before verb, $\boldsymbol{b} \boldsymbol{a}$ has to be used to mark the object to distinguish from the subject as in (9b) instead of (9c), that is, $\boldsymbol{b} \boldsymbol{a}$ becomes obligatory; on the other hand, we can also have (9d) without $\boldsymbol{b} \boldsymbol{a}$ as object marker when the direct object NP has to change from yi-ge ping-guo to ping-guo.It is apparent that direct object can be placed both in preverbal and in postverbal position and object in preverbal position can be preceded with or without $\boldsymbol{b a}$.

Compare the sentences below, in which the object $\boldsymbol{t} \boldsymbol{a}$ is placed post-verbally in (10a) and pre-verbally in (10b). According to (10d) and (10b), we can conclude that when the object $\boldsymbol{t} \boldsymbol{a}$ is moved to preverbal position, $\boldsymbol{t} \boldsymbol{a}$ must be preceded by $\boldsymbol{b} \boldsymbol{a}$, otherwise (10d) is ungrammatical. In other words, omitting the $\boldsymbol{b} \boldsymbol{a}$ marker is impossible for the pronoun object $\boldsymbol{t} \boldsymbol{a}$ in preverbal position. In (10c), compared with (10b), we can observe that the understood subject is not explicitly expressed.

(10) a. Wo qi si ta le

I angry dead s/he ASP

'I made him/her angry to death.'

b. Wo ba ta qi si le

I ba him/her angry dead ASP

c. $B a$ ta qi si le

ba her/him angry dead ASP

d. *Wo ta qi si le

I s/he angry dead ASP 
Compare the sentences (11) below. In (11a) direct object lao-hu is placed post-verbally while in (11b) it is moved to pre-verbal position with $\boldsymbol{b} \boldsymbol{a}$ as obligatory object marker compared with (11d).
a. Ta da si lao-hu le s/he beat dead tiger ASP
b. Ta ba lao -hu da si le s/he ba tiger beat dead ASP
c. ba lao -hu da si le ba tiger beat dead ASP
d. *Ta lao -hu da si le s/he tiger beat dead ASP

The above examples illustrate that $\boldsymbol{b} \boldsymbol{a}$ as an object case marker is "sometimes obligatory, sometimes optional and sometimes prohibited", which is determined by the "word order" and "semantic properties of the object" (Bergen, 2006, p.10).

Firstly, Greenberg (1963) has already observed the relationship between word order and case marking. Based on his vast typological research, he established a number of linguistic universals, including the implicational universal 41: "if in a language the verb follows both the nominal subject and nominal object as the dominant order, the language almost always has case system" (p.96).

It follows that if a language would change its word order from SVO to SOV, it is to be expected that it develops case marking. In Chinese, most of scholars claim that the dominant word order is SVO. In a canonical Chinese sentence, the prototypical syntactic position for objects in Chinese is postverbal position, that is the arguments of subject and object are distinguished by their syntactic position in relation to the verb, so word order already provides the necessary information to discriminate the grammatical roles of the arguments, and in this case $\boldsymbol{b} \boldsymbol{a}$ is never used for the purpose of discrimination (Bergen, 2006, p.35; Song, 2001, p.156) as shown in (9a) and (10a) and (11a). However, when direct objects are scambled preverbally as shown in the sentence (9b) and (10b) and (11b), both the subjects and objects are on the same side of the verb, the word order change from SVO to SOV. In SOV construction, the subjects and objects can no longer be discriminated from each other by means of their position relative to verb. This can be solved by marking either or both the subjects and the objects with case. If the subject or the object receives a case marker, it can be identified regardless of its position in the sentence (Bergen, 2006). That is the reason why (9b) and (10b) and (11b) have to be marked by $\boldsymbol{b} \boldsymbol{a}$. In addition, in these examples $\boldsymbol{b} \boldsymbol{a}$ as object marker is obligatorily. Why? I'll discuss later. Now we can therefore conclude that the use of $\boldsymbol{b} \boldsymbol{a}$ as an object marker in Chinese is licensed by a shift in "word order". As is known, in terms of basic word order SVO, subjects are prototypically in preverbal position, while objects are generally in postverbal position. "When objects scramble, they end up in a atypical position for objects, as a result of which they can no longer be identified as objects by means of their position, relative to the verb" (Bergen, 2006, p.46). In this case, $\boldsymbol{b} \boldsymbol{a}$ as object marker is used. However, $\boldsymbol{b} \boldsymbol{a}$ is not always obligatory, for $\boldsymbol{b} \boldsymbol{a}$ can be omitted under some circumstances. Next I'll investigate what triggers the omission of $\boldsymbol{b} \boldsymbol{a}$ marker.

Secondly, the semantic features of animacy and definiteness or specificity of the object determine whether the objects are marked or not with case marker (Aissen, 2003; Bergen, 2006). For example, in Hebrew indefinite objects are not case marked, in contrast with the definite objects marked; on the other hand in Malayalam human and animate objects are case marked while inanimate objects are not (Asher \& Kumari, 1997; Bergen, 2006). "Nouns can be ranked by animacy or definiteness on a continuum ranging from most to least animate or definite" (Bergen, 2006, p.14), thus forming a universal prominence scale:

Animacy scale: Human > Animate > Inanimate

Definite scale: Pronoun > Proper Noun $>$ Definite NP > Definite specific NP > Indefinite non-specific NP (from Bergen, 2006)

Aissen (2003, p.435) generalize that "the higher in prominence a direct object, the more likely it is to be overtly case marked". We can see from the above examples (9), (10) and (11) this universal prominence scale has influence on the $\boldsymbol{b} \boldsymbol{a}$ as obligatory marker or optional marker. When we compare (10b) with (10d) and (11b) with (11d), we can find that the preverbal objects are human and animate respectively, and $\boldsymbol{b} \boldsymbol{a}$ is retained there, that is, it cannot be omitted. On the other hand, when the preverbal object is inanimate as shown in (9b) with (9d), $\boldsymbol{b} \boldsymbol{a}$ is optional and can be omitted. Therefore the dimension of animacy determines whether $\boldsymbol{b a}$ object marker is obligatory or optional and the cut-off point on the animacy scale is placed between animate and inanimate objects. In terms of universal prominence scale, human and animate are more prominent than inanimate, so it is likely to be ovetly marked according to the generalization that "the higher in prominence a direct object, the more likely it is to be overtly case marked" (Aissen, 2003, p.435). Consequently, the human and animate preverbal objects in (10b) and (11b) are obligatory marked with ba, while inanimate preverbal object in (9d) can be omitted. As is shown in the example (9d), ba as object marker is semantically empty, and only plays a syntactic role, so, it can be omitted without affecting the meaning and grammaticality of the sentence, if the preverbal object is inanimate. In addition, Bergen (2006) argues that "whereas the optional omission of $\boldsymbol{b} \boldsymbol{a}$ can be seen as a consequence of animacy features of the object, the presence of $\boldsymbol{b} \boldsymbol{a}$ in itself is not semantically driven; it is a consequence of the syntactic structure of sentence"(p. 91). However, $\boldsymbol{b} \boldsymbol{a}$ is not optional for every inanimate preverbal object as shown in (9c). 
In order to account for this situation, we have to turn to the dimension of definiteness-definite scale. So the dimension of definiteness becomes applicable, when the object is inanimate. Given the universal prominence scale, when objects are definite, they have high prominence, while objects are indefinite, they have low prominence, so definite objects are more likely to be overtly marked than indefinite objects, according to the generlization made by Aissen (2003, p.435). It follows that $\boldsymbol{b} \boldsymbol{a}$ is obligatory when preverbal objects are definite, while $\boldsymbol{b} \boldsymbol{a}$ is optional when preverbal objects are indefinite. However, when we compare (9b) \& (9c) \& (9e), we can observe that ba is optional in (9e) with definite object, and obligatory in (9b) with indefinite object. As we know, Chinese doesn't have definite or indefinite articles, but $y i-g e$ and zhe-ge represents indefiniteness an definiteness respectively. So, it is true that the omission of $\boldsymbol{b} \boldsymbol{a}$ is determined by the definiteness of object (ping guo), but it is in conflict with the Aissen's generalizaton that the more prominent the object is, the more likely it is overtly marked.

From the above discussion, we can draw the conclusion that the dimensions of animacy and definiteness determine the omission of $\boldsymbol{b a}$ when object is located in preverbal position. If animate or human objects are placed preverbally, they have to be obligatorily marked with $\boldsymbol{b} \boldsymbol{a}$, while $\boldsymbol{b} \boldsymbol{a}$ is omitted if inanimate objects are in preverbal position. Furthermore, if preverbal objects are indefinite, $\boldsymbol{b} \boldsymbol{a}$ is obligatory, while $\boldsymbol{b} \boldsymbol{a}$ can be omitted if they are definite. Finally, I fully agree with Bergen (2006) that “the way in which animacy influences ba fits Aissen's (2003) cross-linguistic predictions: high prominent objects are obligatorily marked, and low prominent objects are optionally marked. However, the way in which definiteness influences $\boldsymbol{b} \boldsymbol{a}$ appears to conflict" (p.48).

\section{CONCLUSION}

The unmarked order of Chinese is SVO. When the word order is changed to SOV, where the object precedes the verb and thus is marked by a special morpheme $\boldsymbol{b} \boldsymbol{a}$, for $\boldsymbol{b} \boldsymbol{a}$ as a marker can be used to distinguish subjects from objects. Under normal circumstances, $\boldsymbol{b} \boldsymbol{a}$ marks the direct object, ${ }^{7}$ but there are apparently cases where $\boldsymbol{b} \boldsymbol{a}$ marks the subject of an intransitive. For this reason, Frei (1956) proposed an ergative analysis of $\boldsymbol{b} \boldsymbol{a}$, thus $\boldsymbol{b} \boldsymbol{a}$ is treated as an absolutive marker. In this paper, based on the criticism made by scholars, I demonstrate that I don't consider $\boldsymbol{b} \boldsymbol{a}$ to be ergative phenomenon. One reason is that some of the cases in which $\boldsymbol{b} \boldsymbol{a}$ marks intransitive subjects are proved to have unexpressed subjects, for these understood subjects can be inferred from the context. Second is that several cases which are said to be true examples turn out to be unacceptable in modern Chinese. Finally, Li and Yip argue that the established cases of ergative languages have ergative marked and absolutive unmarked. If the ergative is zero-marked in Chinese, and absolutive marked by $\boldsymbol{b} \boldsymbol{a}$, this would be the only such case known.

A question arises that how we can account for the $\boldsymbol{b} \boldsymbol{a}$ marker, if it is not absolutive. I propose that $\boldsymbol{b} \boldsymbol{a}$ should be treated only as an object marker, instead of an absolutive marker. However, only when the object is moved to preverbal position, can $\boldsymbol{b} \boldsymbol{a}$ be added to mark the object. In Chinese, since the subject and object are placed on opposite sides of the verb, their grammatical functions are distinguished by their syntactic position. If the word order is changed to SOV with the object preverbally positioned, in this case, word order is no longer enough in distinguishing subjects from objects, so $\boldsymbol{b} \boldsymbol{a}$ is needed to determinate the grammatical roles of the arguments. Under normal circumstances, $\boldsymbol{b} \boldsymbol{a}$ should be obligatorily used. In fact, according to observation, the semantic features of animacy and definiteness or specificity of the object determine whether the objects are marked or not with case marker $\boldsymbol{b} \boldsymbol{a}$, that is, $\boldsymbol{b} \boldsymbol{a}$ is optionally marked or obligatorily marked depending on the animacy and definiteness of objects. If animate or human objects are placed preverbally, they have to be obligatorily marked with $\boldsymbol{b} \boldsymbol{a}$, while $\boldsymbol{b} \boldsymbol{a}$ is omitted if inanimate objects are in preverbal position; if preverbal objects are indefinite, $\boldsymbol{b} \boldsymbol{a}$ is obligatory, while $\boldsymbol{b} \boldsymbol{a}$ can be omitted if they are definite.

I can summarize that it is the syntactic position of direct object that determines whether $\boldsymbol{b} \boldsymbol{a}$ as an object marker can be added. Once the direct object is in preverbal position, semantic features of animacy and definiteness determine whether $\boldsymbol{b a}$ is omitted or not. But last bot not the least, the dimension of definiteness becomes applicable only when the object is inanimate.

\section{REFERENCES}

[1] Aissen. (2003). Differential Object Marking: Iconicity Vs Economy. Natural Language and Linguistic Theory, $21,435-483$.

[2] Bergen, Geertje Van. (2006). To ba or not to ba. Master's Thesis, Radboud University Nijmegen. www.ru.nl/publish/pages/518697/geertje_scriptie_tobaornottoba.pdf, (accessed 15/5/2007).

[3] Frei, Henri. (1956). The Ergative Construction in Chinese: Theory of Pekinese PA. Gengo Kenkyu, 31, 22-50.

[4] Fujii, Fumio. (1989). The Typological Status of Chinese and Implications. München: Ludwig-Maximilians Universität.

[5] Greenberg, J.H. (1963). Some Universals of Grammar with Particular Reference to the Order of Meaningful Elements. In J. H. Greenberg (Ed.), Universls of Language. Cambridge: MIT Press, 73-113.

[6] Li, C.N. and S. Thompson. (1981). Mandarin Chinese: A Functional Reference Grammar, Berkeley: University of California Press.

[7] Li, C.N. and S.A. Thompson. (1978). An Exploration of Mandarin Chinese. In Lehmann (Ed.), The Typology of Language. Austin: University of Texas Press, 223-266.

[8] Li, C.N.ed. (1975). Word Order and Word Order Change, Austin: University of Texas Press.

\footnotetext{
${ }^{7}$ There are, of course, many well-known cases of locatives, etc marked by $\boldsymbol{b} \boldsymbol{a}$. It has been argued that these are direct objects (Thompson, 1973).
} 
[9] Li, C.N. and S.A. Thompson. (1974). Historical change of word order: A case study in Chinese and Implications. In Anderson \& Jones (Eds.), Historical Linguistics (vol. A). Amsterdam: North-Holland Publishing Co., 199-217.

[10] Li, C.N. and S.A. Thompson. (1974). An Explanation of Word Order Change SVO-SOV. Foundations of Language, 12, $202-$ 214.

[11] Li, C.N. and S.A. Thompson. (1975). The Semantic Function of Word Order: A case study in Mandarin. In Charles N. Li, (Ed.), Word Order and Word Order Change. Austin: University of Texas Press, 163-195.

[12] Li, C.N. and S.A. Thompson. (1976). Subject and Topic: A New Typology of Language. In Charles N. Li, (Ed.), Subject and Topic. New York: Academic Press, 458-489.

[13] Li, Y. H. (2001). The ba-construction. www.usc.edu/dept/LAS/ealc/chinling/articles/ba1.pdf(accessed 15/5/2007).

[14] Li, Y.C. and Yip, M. (1979). Ba Construction and Ergativity in Chinese. In Plank, F. (Ed.), Ergativity: Towards a theory of grammatical relations. London and New York: Academic Press, 103-114.

[15] Liu, F. (1997). An Aspectual Analysis of ba. Journal of East Asian Linguistics, 6, 51-99.

[16] Plank, F. (1979). Ergativity: Towards A Theory of Grammatical Relations. London and New York: Academic Press.

[17] Plank, Frans. (1995). Research into Syntactic Change III: Ergativity. In J.Jacobs, A. von Stechow, W. Sternefeld and T.Vennemann (Eds), Syntax: An International Handbook of Contemporary Research (vol.2). Berlin: Walter de Gruyter, 11841199.

[18] Silver, Elisabeth. (1989). Ergativity and VSO Word Order: A Configurational Analysis. Working Paper.

[19] Song, Jae Jung. (2001). Linguistic Typology: Morphology and Syntax. Harlow and London: Pearson Education.

[20] Sun, Chaofen. (2006). Chinese: A Linguistic Introduction. Cambridge, England: Cambridge University Press.

Lei Liu was born in Changchun, China in 1976. She received her M.A. degree in English Language and Literature from Jilin University, China in 2005.

She is currently a lecturer in the School of Foreign Languages, Changchun University of Science and Technology, Changchun, China. Her research interests include cognitive linguistics and literature. 